Vol. 2, No. 3, pp. 190-201, (September2021)

DOI: 10.21608/aujes.2021.89249.1034

Aswan University Journal of Environmental Studies (AUJES)

Online ISSN: 2735-4237, Print ISSN: 2735-4229

Journal homepage: https://aujes.journals.ekb.eg/

E-mail: AUJES@aswu.edu.eg

Original research

\title{
Water-Saving from Rehabilitation of Irrigation Canals Case Study: El-Sont
} Canal, Assiut Governorate

\author{
Ashour M. A ${ }^{(1)}$; Tarek Sayed ${ }^{(2)^{*}}$; AbdAllah A.A ${ }^{(3)}$ \\ (1) Prof. of Irrigation Engineering and Water Structures Civil Eng. Dept., Assiut University, \\ ${ }^{(2)}$ Assis. Prof. of Irrigation Engineering and Water Structures Civil Eng. Dept., Assiut University \\ (3) Assis. Lecturer of Irrigation Engineering and Water Structures Civil Eng. Dept., Assiut University.
}

Received: $1 / 8 / 2021$

Accepted: $17 / 8 / 2021$

(C) Unit of Environmental Studies and Development, Aswan University

\begin{abstract}
:
In light of the national project for rehabilitation and the lining of the exposed irrigation canals which is currently under implementation all over Egypt, a field study is introduced to assess the technical, and environmental expected impacts that must be achieved in such a national project. In arid and semi-arid regions, with permeable soils like Egypt, water seeps significantly through the exposed canals cross-sections, in addition to the quantities lost by evaporation under high temperatures. Saving such tangible quantities of water became the most important goal that can be achieved to solve the problematic situation of irrigation water shortage that faces Egypt nowadays. This study is a field attempt to estimate the transmission losses in the El-Sont canal in Middle Egypt (Assuit Governorate) as a representative open channel for a specific type of soil, climate, topography, and beneficiary's lifestyle. The produced and developed equations introduced and recommended by the most popular authors in this field were studied, summarized, and presented in a tabulated form to be easily used, and for comparison purposes. Using the recommended equations developed by the most popular reviewed researchers, the conveyance and transmission losses for the understudy El-Sont canal, and its off-taking canals were calculated and evaluated to be about $248628 \mathrm{~m}^{3} /$ day, i.e., more than $39.54 \mathrm{Mm}^{3} /$ year. That big quantity of saved or recovered water can be used for reclamation and irrigation of about five thousand new acres in the nearby area. The study and the field trips, visits, and the personal direct communication with the farmers and beneficiaries proved that the project had responded to their hopes for an improved living standard, health, in addition to the environmental situation for all the Egyptian countryside.
\end{abstract}

Keywords: Irrigation water saving - Canals rehabilitation - Canals transmission losses

Corresponding author*: E-mail address: tareksayed1986@aun.edu.eg 


\section{Abbreviations :}

The following symbols are used in this paper:

$\begin{array}{llllll}\text { a } & \text { Area of wetted perimeter } & \left(\mathrm{M} \mathrm{m}^{2}\right) ; & \mathrm{L} & \text { Canal length } & (\mathrm{m}) ; \\ \mathrm{B} & \text { Bottom width } & (\mathrm{m}) ; & \mathrm{P} & \text { Wetted perimeter } & (\mathrm{m}) ; \\ \mathrm{C} & \text { Constant depends on soil type } & -; & \mathrm{Q} & \text { Discharge } & \left(\mathrm{m}^{3} / \mathrm{s}\right) ; \\ \mathrm{C}_{1} & \text { Seepage factor }=\left(2 \mathrm{e} / \mathrm{v}^{*} \mathrm{H}\right) & \left(\mathrm{m}^{-1}\right) ; & \mathrm{R} & \text { Hydraulic radius } & (\mathrm{m}) ; \\ \mathrm{C}_{2} & \text { Seepage factor }=\mathrm{e}^{*} \mathrm{~B} & \left(\mathrm{~m}^{2} / \mathrm{s}\right) ; & \mathrm{R}_{\mathrm{E}} & \text { Evaporation losses } & \left(\mathrm{m}^{3} / \mathrm{day}\right) ; \\ \mathrm{C}_{3} & \text { Seepage factor }=2 \mathrm{e}^{*} \mathrm{H}\left(\left(\mathrm{z}^{2}+1\right)^{0.5}-\mathrm{z}\right) & \left(\mathrm{m}^{2} / \mathrm{s}\right) ; & \mathrm{S} & \text { Seepage losses } & \left(\mathrm{m}^{3} / \mathrm{s} / \mathrm{km}\right) ; \\ \mathrm{E} & \text { Evaporation rate } & (\mathrm{m} / \mathrm{day}) ; & \mathrm{T} & \text { Width of canal water surface } & (\mathrm{m}) ; \\ \mathrm{e} & \text { Permeability } & (\mathrm{m} / \mathrm{s}) ; & \mathrm{v} & \text { Mean velocity } & (\mathrm{m} / \mathrm{s}) ; \\ \mathrm{H} & \text { Water depth } & (\mathrm{m}) ; & \mathrm{z} & \text { Canal side slope } & -; \text { and } \\ \mathrm{i} & \text { Bed slope } & -; & \alpha & \text { Seepage losses factors } & -.\end{array}$

\section{1- INTRODUCTION}

In Egypt, open channels are the major conveyance system of irrigation water from its sources to where it may need through a total length exceeding $32000 \mathrm{~km}$ (El Gamal and Zaki, 2017). This network was excavated in the sedimentary permeable soils and running in hot weather conditions. A huge amount of water may be lost through more than one aspect, such as seepage, evaporation, evapotranspiration, and other bad behaviors in using the irrigation water for other purposes. As a compatible scientific-technical role of the university, this field study was conducted on one of the under-rehabilitation process canals in the Assuit countryside in the Middle Egypt region, to be a representative case study. About the rehabilitation and environment, many field trips were carried out through which the environmental condition of the understudy canal and its off-takings surrounding areas were recorded. The recorded observations included all the basic services and infrastructure related to the rehabilitation national project which were evaluated to assess the gained positive needed impacts.

\section{2- LITERATURE REVIEW}

The major types of losses in irrigation canals are the seepage losses through the canal sides and bed, the evaporation losses from the free surface of the canal top width, and the transpiration losses from grass and weeds that located at the side slope, bed of canals, or floating weeds. Other types such as leakage (water lost through channel banks and structures) and operational losses (outfalls, system fill, unmetered diversions, and inaccurate metering), (Allen et al., 1998; Kacimov, 1992; Swamee, 2000; Akkuzu, 2012; Ahuchaogu, 2015). Although the transpiration, leakage, and operational losses may be very high in some instances, these losses are out of the scope of this study. Two main types of water losses will be discussed in this paper, namely losses due to seepage and evaporation. The seepage losses in the irrigation canals account for about $98.37 \%$ of the water conveyance losses, while approximately $0.3 \%$ of the total stream is lost due to evaporation (Jadhav, 2014).

\subsection{Seepage Losses}

A review of the literature confirmed that seepage rates from canals are greatly affected by the permeability of canal soil, canal wetted perimeter length and shape, canal water depth, uniformity of the soil or canal lining, flow rate, sediment load, and size distribution, canal age, groundwater table location, and constraints on groundwater flow, presence of wells, rivers, drains and impermeable boundaries, etc. Other relatively less significant factors like viscosity, the salinity of 
water, aquatic plants, operational time, water chemistry, and temperature (Swamee, 2000; Alam, 2004). According to Zhang (2017) seepage has many impacts on canals' water quantity and quality. Seepage losses can account for $20-30 \%$ of the total flow volume in unlined earthen canals (Martin, 2015). Seepage rate can be evaluated by three methods: direct measurement methods (field measurements), analytical equations, and empirical formulas (Robinson and Rohwer 1957). Field tests for measuring seepage such as inflow-outflow measurement method, ponding tests, point measurement method, double-ring infiltration test, and parameter measurement method (Alam, 2004). There are different empirical and analytical equations used to evaluate the seepage losses. Mowafy (2001) evaluated several empirical and analytical formulas with seepage tests performed on different sections of the Ismailia canal in Egypt. The results showed that the Molesworth-Yennidumia empirical formula (Shaikh, 2016) as follows in Eq. 1, had good agreement with the field results.

$$
\mathrm{S}=\mathrm{CLP} \sqrt{\mathrm{R}}
$$

Where: $S$ is seepage $\left(\mathrm{m}^{3} / \mathrm{s} / \mathrm{km}\right) ; L$ is the canal length $(\mathrm{m}), P$ is wetted perimeter $(\mathrm{m}) ; R$ is hydraulic radius, $C$ is constant whose value depends on the type of soil and was taken to be equal to $0.003,0.0015,0.0018,0.0022$, and 0.0026 for sandy loam, clay, silty clay, clay loam, and silty loam, respectively (Shaikh, 2016; Awan, 2017).

International commission on irrigation and drainage developed another equation called the Indian formula which used for estimating the earthen canals seepage as follows (Khan, 2015):

$$
\mathrm{S}=\mathrm{CaH}
$$

Where $S$ is the total seepage losses $\left(\mathrm{ft}^{3} / \mathrm{s}\right)$; a is the area of wetted perimeter (million $\left.f t^{2}\right)$; $H$ is the depth (ft); and $C$ is a factor which depends on soil types and varies from 1.1 to 1.8.

Moritz formula was proposed by the United States Bureau of Reclamation (USBR) for estimating seepage losses per mile of the unlined canal as follows (Kraatz, 1977; Leigh, 2014):

$$
\mathrm{S}=0.2 \times \mathrm{C} \times \sqrt{\mathrm{Q} / \mathrm{V}}
$$

Where $S$ is the seepage losses in (ft /sec/mile), $Q$ is the discharge (ft / / ec), $V$ is the mean velocity (ft/sec), and $C$ is a constant value depending on soil type. (cemented gravel and hard pan with sandy loam 0.34, clay and clayey loam 0.41, sandy loam 0.66, sandy soil with rock 1.68, sandy and gravelly soil 2.20)

Mowafy (2001) reviewed that Molesworth and Yennidunia derived analytical formula to estimate seepage losses as follows:

$$
\mathrm{S}=\frac{\alpha * 10^{-4}}{\mathrm{R}^{1.166 * * 0.5}}
$$

Where $S$ is the seepage losses in $\mathrm{m}^{3} / \mathrm{s} / \mathrm{km} ; R$ is the hydraulic radius. $i$ is the bed slope, $\alpha$ is seepage losses factors equal to $0.375 \mathrm{~m}^{-1}$ for clay soil, and $0.75 \mathrm{~m}^{-1}$ for sandy soil.

Farouk (1979) developed an analytical equation to estimate seepage losses in the earthen canal as given below:

$$
\mathrm{S}=\mathrm{Q}\left[\frac{\mathrm{e}^{\mathrm{C}_{1} \mathrm{~L}}-1}{\mathrm{e}^{\mathrm{C}_{1} \mathrm{~L}}}\right]-\mathrm{L}\left(\mathrm{C}_{2}-\mathrm{C}_{3}\right) / \mathrm{e}^{\mathrm{C}_{1} \mathrm{~L} / 2}
$$

Where $S$ is the seepage losses from canal in $\mathrm{m}^{3} / \mathrm{sec} ; Q$ is the flow discharge in $\mathrm{m}^{3} / \mathrm{sec}$; $e_{2}$ is the permeability in $\mathrm{m} / \mathrm{sec}$; $L$ is length of canal in $\mathrm{m}$; Vis average velocity in $\mathrm{m} / \mathrm{sec}$; $D$ is the water depth in $m ; B$ is the bottom width $m$; and $z$ is the side slope, $C_{1}=\left(2 e_{2} / v . d\right)$ : the seepage factor in $\mathrm{m}^{-1} ; C_{2}=e_{2} b$ : the seepage factor $\mathrm{m}^{2} / \mathrm{sec} ; C 3=2 e_{2} d\left(\left(z^{2}+1\right)^{0.5}-z\right)$ : the seepage factor $\mathrm{m}^{2} / \mathrm{sec}$. 
Davis-Wilson formula (Eq. 6)_relates seepage losses directly to the cube root of the water height in the canal and considers infiltration to be equal around the wetted perimeter. Davis-Wilson equation was cited for estimating seepage losses for lined canals, but also provided constant values for an array of soil types (Kraatz, 1977; Leigh, 2014):

$$
\mathrm{S}=\mathrm{C} \frac{\mathrm{P} \times \mathrm{L} \times \mathrm{H}^{1 / 3}}{4 \times 10^{6}+2000 \sqrt{\mathrm{v}}}
$$

Where: $S$ is seepage losses $\left(\mathrm{ft}^{3}\right.$ per second per length of canal); L is the length of canal (ft); $P$ is the wetted perimeter $(f t) ; H$ is the mean water depth in the canal $(f t) ; v$ is the velocity of flow in the canal (ft/sec); and $C$ are the constant values depending on lining (values of $C=1$ concrete ( 3 to 4 inches thick), 4 clay puddle or mass clay (6 inches thick), 5 thick new coat of crude oil or light asphalt, 6 cement plaster (1 inch thick), 8 clay puddle (3 inches thick), 10 cement grout or asphalt, 12 clay soil, 15 clay loam soil, 20 Medium loam, 25 Sandy loam, 40 Fine sand, (Leigh, 2014).

Mowafy (2001) advised of using all analytical equations and the empirical formula of Molesworth and Yennidunia for computing seepage losses in all canals of Egypt. So, in this paper, the empirical formula of Molesworth and Yennidunia will be used for computing seepage losses in El-Sont canal and its network. Also, for increasing the accuracy of the results, the Indian equation and Mortez's equation will be used.

\subsection{Evaporation Losses}

In hot weather conditions, when the canal water is exposed to the atmosphere, the surface water transfer from the liquid stage to the vapor stage. The evaporation losses are very small compared to the seepage losses. It may range from 0.25 to $1 \%$ of the total canal discharge (Sahasrabudhe, 2011). Commonly, there are many methods used for estimating evaporation loss from canals like the evaporation pan, the water balance method, the heat balance method (the energy balance method), and the aerodynamic method which given by Liu (2016) and Wang (2019). The rate of evaporation from a running water surface depended not only on the surface wind speed but also on the flow speed. In the evaporation pan test, the evaporation rate from pans filled with water is easily obtained. In the absence of rain, the amount of water evaporated during a period ( $\mathrm{mm} /$ day) corresponds to the decrease in water depth in that period. Pans provide a measurement of the integrated effect of radiation, wind, temperature, and humidity on the evaporation from an open water surface. According to Liu (2016) the evaporation losses of water in irrigation canals $\left(\mathrm{R}_{\mathrm{E}}\right)$ from the intake to the fields through total canal length is estimated by the following equation:

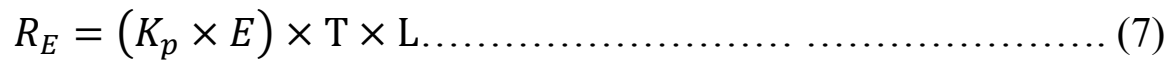

In which $K_{p}$ is the pan coefficient; $E$ is the evaporation rate ( $m /$ day); $T$ is the width of canal water surface $(m)$; L is the total canal length $(m)$.

\section{3- MATERIALS AND METHODS}

\section{1- Description of the study area}

The present study was conducted on the El-Sont canal as one of the under-rehabilitation canals in Assuit countryside in the Middle Egypt region, as a case study. The length of the canal is about $40 \mathrm{~km}$. It takes its water at km 157 from Eastern Nag-Hamadi main canal. It serves an area of about 34040 acres, distributed between Abnoub (23550 acres), and Sahel-Seleem (10490 acres). Both belong to the irrigation engineering administration in Assiut City. Twenty-four off-taking canals are branched directly from the El-Sont canal, with a network of 58 off-taking canals as 
shown in Fig. 1. Canals network is divided into three watering shifts (five days' work and 10 days off). The total length of the off-taking canals is about $149.16 \mathrm{~km}$ (MWRI, 2020). The area is characterized by an arid climate. The geometrical dimensions for the designed water sections of the El-Sont canal and its network are presented in Table 1.

\section{2- Soil type}

For knowing, the soil along the path of the canal, it was necessary to take some samples to determine the type of soil, its nature, and degree of permeability. Soil samples were taken every five $\mathrm{km}$ along the canal at different depths under the bed of the canal and from the side slopes. Also, the different parameters and coefficients which are used in the empirical equations concerning the study were determined.

\section{3- Seepage losses computations}

The estimated quantities of water lost by seepage for both the designed, and the existing sections of the El-Sont canal and its network, were estimated using the Egyptian formula (Eq. 1) as recommended by Mowafy (2001). At the same time, for the accuracy purposes, such quantities were calculated also using the Indian formula (Eq. 2) and Mortiz formula (Eq. 3). The work was carried out for reaches of $300 \mathrm{~m}$. long, for the under-study canals. The results of such calculations are summarized in Table 2.

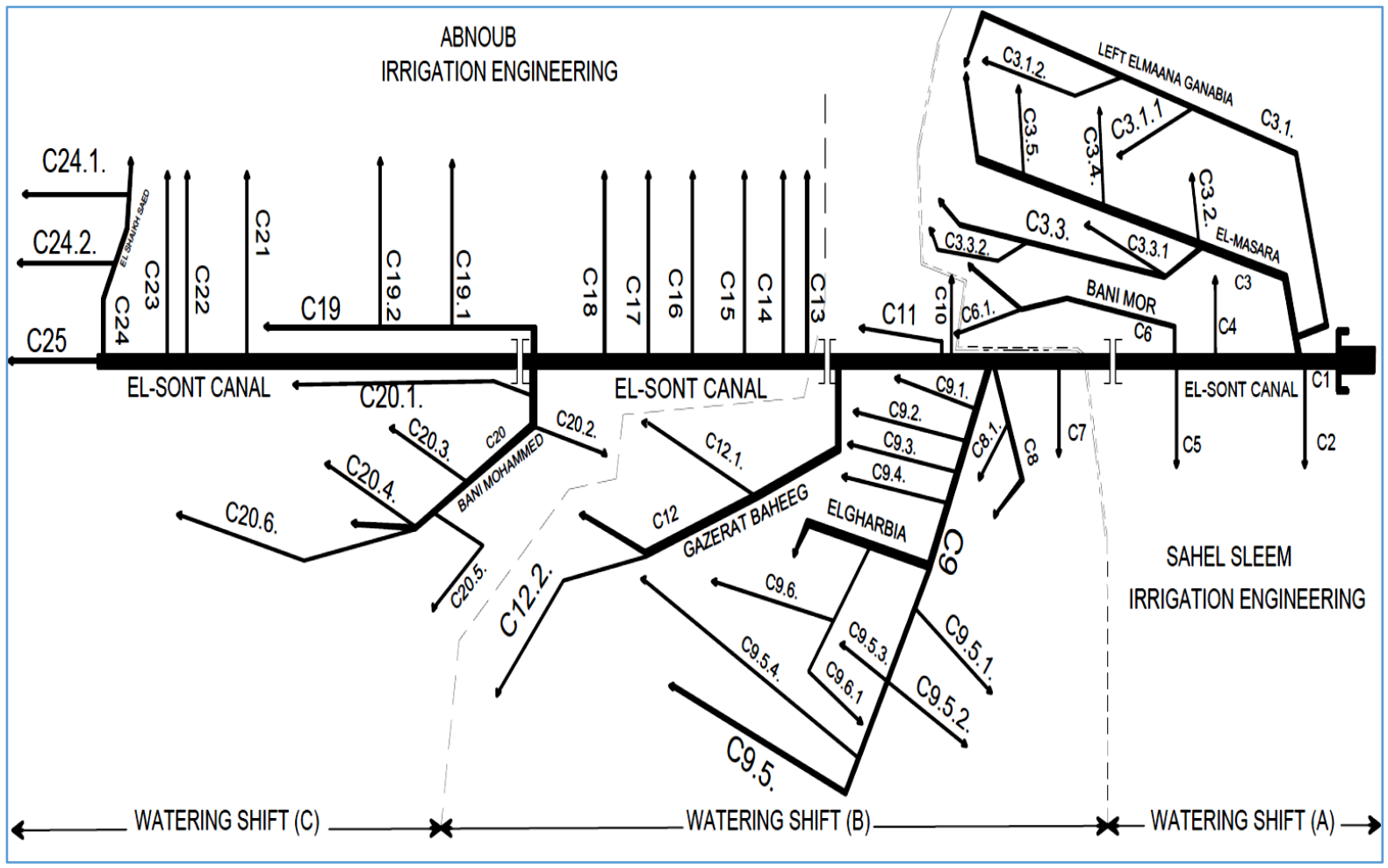

Fig. 1. El-Sont canal and its network.

\section{4- Evaporation losses computation}

Using the meteorological data of the study area according to the recorded sheets of ArabAlawamer official meteorological station which is shown in Table 3, the evaporation losses were calculated using (Eq. 7). The values of maximum evaporation losses for both the designed and existing water sections of El-Sont canal and its network were calculated for reaches $300 \mathrm{~m}$. long and summarized in Table 4. 
Aswan University Journal of Environmental Studies (AUJES) 2 (3), pp. 190-201, (2021).

Online ISSN: 2735-4237, Print: ISSN 2735-4229. https://aujes.journals.ekb.eg/

Table 1: Geometric dimensions for the designed water sections of El-Sont canal and its network, (MWRI, 2020).

\begin{tabular}{|c|c|c|c|c|c|c|c|}
\hline \multirow[b]{2}{*}{ No } & \multirow[b]{2}{*}{ Canal Name } & \multicolumn{2}{|c|}{ Section } & \multirow{2}{*}{$\begin{array}{c}\text { Bed } \\
\text { Width } \\
(\mathbf{m}) \\
\end{array}$} & \multirow[b]{2}{*}{ Side Slope } & \multirow{2}{*}{$\begin{array}{c}\text { Water } \\
\text { Depth } \\
\text { (m) }\end{array}$} & \multirow{2}{*}{$\begin{array}{c}\text { Discharg } \\
\left(\mathbf{m}^{3} / \mathbf{s}\right)\end{array}$} \\
\hline & & $\begin{array}{c}\text { From } \\
\text { Km }\end{array}$ & $\begin{array}{c}\text { To } \\
\text { Km } \\
\end{array}$ & & & & \\
\hline \multirow{6}{*}{1} & El-Sont (sahel sleem) & 0 & 7.6 & 12 & $3: 2$ & 2.6 & 21.8 \\
\hline & \multirow{5}{*}{ El-Sont (abnoub) } & 7.6 & 15.94 & 11 & $3: 2$ & 2.6 & 17.62 \\
\hline & & 15.94 & 23.2 & 9 & $3: 2$ & 2.6 & 17.62 \\
\hline & & 23.2 & 26.4 & 8 & $3: 2$ & 2.35 & 15.33 \\
\hline & & 26.4 & 34.12 & 7 & $3: 2$ & 1.9 & 6.64 \\
\hline & & 34.12 & 39.9 & 5 & $3: 2$ & 1.4 & 5.76 \\
\hline 2 & Sothern Elnabary & 0 & 3.2 & 1 & $1: 1$ & 1 & 0.35 \\
\hline \multirow{3}{*}{3} & \multirow{3}{*}{ Elmaasara } & 0 & 3.6 & 4 & $1: 1$ & 2.4 & 6.76 \\
\hline & & 3.6 & 6.98 & 3 & $1: 1$ & 1.75 & 3.98 \\
\hline & & 6.98 & 8.8 & 2 & $1: 1$ & 1.5 & 1.64 \\
\hline \multirow{3}{*}{3.1} & \multirow{3}{*}{ Left Elmaana Ganabia } & 0 & 5 & 3 & $1: 1$ & 1.85 & 2.7 \\
\hline & & 5 & 8.8 & 2 & $1: 1$ & 1.6 & 2.35 \\
\hline & & 8.8 & 10.45 & 1 & $1: 1$ & 1.15 & 0.82 \\
\hline 3.1 .1 & Serage Banch & 0 & 2 & 1 & $1: 1$ & 0.8 & 0.35 \\
\hline 3.1 .2 & Elfaiama Branch & 0 & 4 & 1 & $1: 1$ & 1 & 0.71 \\
\hline 3.2 & Amro Branch & 0 & 2.4 & 1 & $1: 1$ & 0.9 & 0.27 \\
\hline \multirow{4}{*}{3.3} & \multirow{4}{*}{$\begin{array}{l}\text { Salebat Elmaasara } \\
\text { Ganabia }\end{array}$} & 0 & 1.3 & 4 & $1: 1$ & 1.4 & 1.52 \\
\hline & & 1.3 & 4.34 & 3 & $1: 1$ & 1.18 & 1.25 \\
\hline & & 4.34 & 5.4 & 2.5 & $1: 1$ & 1.1 & 0.85 \\
\hline & & 5.4 & 6.7 & 2 & $1: 1$ & 1 & 0.5 \\
\hline 3.3 .1 & Elmanshia Branch & 0 & 1.4 & 1 & $1: 1$ & 0.8 & 0.27 \\
\hline 3.31 & Elgamasea Branch & 0 & 2.1 & 1 & $1: 1$ & 0.9 & 0.39 \\
\hline 3.4 & Elqasr Branch & 0 & 1.14 & 1 & $1: 1$ & 0.9 & 0.27 \\
\hline 3.5 & Elquata Branch & 0 & 1.36 & 1 & $1: 1$ & 0.75 & 0.26 \\
\hline 4 & Elghwaish Branch & 0 & 1.3 & 1 & $1: 1$ & 0.75 & 0.21 \\
\hline 5 & Elnabari Alwasta & 0 & 2.45 & 1 & $2: 1$ & 1 & 0.35 \\
\hline \multirow{2}{*}{6} & \multirow{2}{*}{ Bani Mor } & 4.5 & 5.2 & 3 & $1: 1$ & 1.5 & 2.04 \\
\hline & & 5.2 & 6.3 & 2 & $1: 1$ & 1 & 1 \\
\hline 6.1 & Quernaw Branch & 0 & 1.42 & 2 & $1: 1$ & 0.75 & 0.27 \\
\hline 7 & Gazerat Bani Mor & 0 & 1.6 & 1.5 & $3: 2$ & 1 & 0.4 \\
\hline 8 & Sahel Bani Mor & 0 & 2.65 & 1.5 & $2: 1$ & 1 & 0.73 \\
\hline 8.1 & Elbaharwa & 0 & 1.3 & 1 & $1: 1$ & 0.75 & 0.15 \\
\hline \multirow{2}{*}{9} & \multirow{2}{*}{ Elgharbia } & 0 & 2 & 4 & $3: 2$ & 1.75 & 4.62 \\
\hline & & 2 & 5.99 & 2.5 & $1: 1$ & 1 & 2.47 \\
\hline 9.1 & Hoshet Kom Aboshil & 0 & 1.65 & 1 & $1: 1$ & 0.75 & 0.1 \\
\hline 9.2 & Ali Bek & 0 & 2.1 & 1 & $1: 1$ & 0.9 & 0.35 \\
\hline 9.3 & Western Elnasara & 0 & 3.75 & 2 & $1: 1$ & 1.65 & 0.85 \\
\hline 9.4 & Eastern Elnasara & 0 & 3 & 2 & $1: 1$ & 1.7 & 0.85 \\
\hline 9.5 & Hablass & 0 & 5 & 2 & $1: 1$ & 1.4 & 1 \\
\hline
\end{tabular}

\begin{tabular}{|c|c|c|c|c|c|c|c|}
\hline \multirow[b]{2}{*}{ No } & \multirow[b]{2}{*}{ Canal Name } & \multicolumn{2}{|c|}{ Section } & \multirow{2}{*}{$\begin{array}{c}\text { Bed } \\
\text { Width }(m)\end{array}$} & \multirow[b]{2}{*}{ Side Slope } & \multirow{2}{*}{$\begin{array}{c}\text { Water } \\
\text { Depth } \\
\text { (m) }\end{array}$} & \multirow{2}{*}{$\begin{array}{c}\text { Discharge } \\
\left(\mathrm{m}^{3} / \mathbf{s}\right)\end{array}$} \\
\hline & & $\begin{array}{c}\text { From } \\
\mathbf{K m}\end{array}$ & To Km & & & & \\
\hline 9.5 .1 & Elakrad Branch & 0 & 1.8 & 1 & $1: 1$ & 1 & 0.17 \\
\hline 9.5 .2 & Bani Zeid Branch & 0 & 2 & 1.5 & $1: 1$ & 0.85 & 0.22 \\
\hline 9.5 .3 & Diab Branch & 0 & 0.9 & 1 & $1: 1$ & 0.9 & 0.1 \\
\hline 9.5 .4 & Hablas Elgadida & 0 & 4.8 & 2 & $1: 1$ & 1.58 & 1.02 \\
\hline 9.6 & Hoshet Eltwabia & 0 & 1.1 & 2 & $1: 1$ & 1.1 & 0.47 \\
\hline 9.6 .1 & Eltwabia Southern Branch & 0 & 2.25 & 2 & $1: 1$ & 1 & 0.27 \\
\hline 10 & Kom Abo Shail Branch & 0 & 1 & 1 & $1: 1$ & 0.9 & 0.4 \\
\hline 11 & Right Southern Elsont Ganabiat & 0 & 3 & 2 & $1: 1$ & 0.85 & 0.6 \\
\hline \multirow{3}{*}{12} & \multirow{3}{*}{ Baheege } & 0 & 2.4 & 4 & $3: 2$ & 1.5 & 2.71 \\
\hline & & 2.4 & 4.5 & 3 & $3: 2$ & 0.9 & 2.1 \\
\hline & & 4.5 & 6.3 & 2 & $3: 2$ & 0.75 & 0.4 \\
\hline 12.1 & Abo Amera & 0 & 1.9 & 2 & $1: 1$ & 1.4 & 0.55 \\
\hline 12.2 & Elkhalifaa & 0 & 1 & 1.5 & $1: 1$ & 1.2 & 0.35 \\
\hline 13 & Bani Rezah & 0 & 2.6 & 2 & $1: 1$ & 1.1 & 0.42 \\
\hline 14 & Abnoub Branh & 0 & 2 & 1 & $1: 1$ & 0.75 & 0.47 \\
\hline 15 & Elkadadeh Western Branch & 0 & 2.2 & 1.5 & $1: 1$ & 1 & 0.93 \\
\hline 16 & Bani Ibrahem Western Branch & 0 & 2.3 & 1 & $1: 1$ & 1.1 & 0.47 \\
\hline 17 & Elsawalem Southern Branch & 0 & 2 & 1 & $1: 1$ & 0.95 & 0.6 \\
\hline 18 & Elrawateb Branch & 0 & 1.25 & 1 & $1: 1$ & 0.85 & 0.38 \\
\hline \multirow{2}{*}{19} & \multirow{2}{*}{ Right Northern Elsont Ganabiat } & 0 & 3.3 & 4 & $3: 2$ & 1.5 & 3.25 \\
\hline & & 3.3 & 5.7 & 2 & $3: 2$ & 1.15 & 1.7 \\
\hline 19.1 & Elsihabia Branch & 0 & 2.05 & $\overline{1}$ & $3: 2$ & 1.3 & 1 \\
\hline 19.2 & Asham Allah & 0 & 1.6 & 1 & $3: 2$ & 1 & 0.5 \\
\hline \multirow{3}{*}{20} & \multirow{3}{*}{ Bani Mohamed } & 0 & 0.38 & 5 & $3: 2$ & 1.45 & 4.47 \\
\hline & & 0.38 & 3.7 & 4 & $3: 2$ & 1.3 & 3.34 \\
\hline & & 3.7 & 6.4 & 3 & $3: 2$ & 1.2 & 2.09 \\
\hline 20.1 & Left Northern Elsont Ganabiat & 0 & 2.55 & 2 & $1: 1$ & 1 & 0.79 \\
\hline 20.2 & Elmarwna & 0 & 1.1 & 1.5 & $1: 1$ & 1 & 0.23 \\
\hline 20.3 & Sahel Elaqab & 0 & 1.3 & 1 & $3: 2$ & 1 & 0.31 \\
\hline 20.4 & Abo Diab Branch & 0 & 1.7 & 1 & $1: 1$ & 1 & 0.63 \\
\hline 20.5 & Elmansora Western Branh & 0 & 1 & 1 & $1: 1$ & 0.85 & 0.4 \\
\hline 20.6 & Sahel Bani Mohammed & 0 & 4.5 & 2.5 & $3: 2$ & 0.9 & 1.04 \\
\hline 21 & Shaqequel & 0 & 2 & 2.5 & $1: 1$ & 1.1 & 0.88 \\
\hline 22 & Elmaabda Sothern Branch & 0 & 1.25 & 1 & $1: 1$ & 1 & 0.5 \\
\hline 23 & Elmaabda Northern Branch & 0 & 2.8 & 2 & $1: 1$ & 1.25 & 1.5 \\
\hline 24 & Elshikh Saed Branh & 0 & 1.2 & 3 & $1: 1$ & 1.2 & 1.88 \\
\hline 24.1 & Abo Meshel & 0 & 1.1 & 1 & $1: 1$ & 0.75 & 0.28 \\
\hline 24.2 & Sahel Elmaabda & 0 & 2.3 & 2 & $1: 1$ & 0.75 & 0.76 \\
\hline 25 & Emtedad Elsont & 0 & 2 & 2 & $3: 2$ & 1.3 & 0.85 \\
\hline
\end{tabular}


Aswan University Journal of Environmental Studies (AUJES) 2 (3), pp. 190-201, (2021).

Online ISSN: 2735-4237, Print: ISSN 2735-4229. https://aujes.journals.ekb.eg/

Table 2: Seepage losses for designed and existing water sections of El-Sont canal and its network $\left(\mathrm{m}^{3} / \mathrm{day}\right)$

\begin{tabular}{|c|c|c|c|c|c|c|c|c|c|c|c|c|c|c|c|}
\hline \multirow{2}{*}{ No } & \multirow{2}{*}{ Canal Name } & \multicolumn{3}{|c|}{ Designed section } & \multicolumn{3}{|c|}{ Existing section } & \multirow{2}{*}{ No } & \multirow{2}{*}{ Canal Name } & \multicolumn{3}{|c|}{ Designed section } & \multicolumn{3}{|c|}{ Existing section } \\
\hline & & Egyptian & Indian & Mortiz & Egyptian & Indian & Mortiz & & & Egyptian & Indian & Mortiz & Egyptian & Indian & Mortiz \\
\hline \multirow{7}{*}{1} & \multirow{2}{*}{ El-Sont (sahel sleem) } & \multirow{2}{*}{29279} & \multirow{2}{*}{40141} & \multirow{2}{*}{19986} & \multirow{2}{*}{29398} & \multirow{2}{*}{49146} & \multirow{2}{*}{18481} & 9.5 & Hablass & 1504 & 2028 & 1570 & 3517 & 3833 & 4230 \\
\hline & & & & & & & & 9.5 .1 & Elakrad Branch & 646 & 655 & 1041 & 522 & 377 & 875 \\
\hline & \multirow{5}{*}{ El-Sont (abnoub) } & 30366 & 41989 & 21231 & 21670 & 24543 & 16225 & 9.5 .2 & Bani Zeid Branch & 724 & 631 & 1156 & 684 & 362 & 942 \\
\hline & & 23358 & 32963 & 17197 & 30110 & 48338 & 20741 & 9.5 .3 & Diab Branch & 287 & 273 & 481 & 117 & 58 & 238 \\
\hline & & 8760 & 11773 & 6811 & 9233 & 13684 & 6961 & 9.5 .4 & Hablas Elgadida & 3763 & 4663 & 4669 & 3517 & 3833 & 4230 \\
\hline & & 16108 & 19308 & 13660 & 12729 & 16160 & 11738 & 9.6 & Hoshet Eltwabia & 1183 & 1116 & 1683 & 1503 & 1093 & 1898 \\
\hline & & 7486 & 7727 & 7453 & 9064 & 11964 & 8839 & 9.6 .1 & Eltwabia Southern Branch & 1110 & 1033 & 1594 & 324 & 211 & 525 \\
\hline 2 & Sothern Elnabary & 1148 & 1164 & 1851 & 2196 & 2911 & 2698 & 10 & Kom Abo Shail Branch & 319 & 303 & 535 & 724 & 662 & 1028 \\
\hline \multirow{4}{*}{3} & \multirow{4}{*}{ Elmaasara } & 6006 & 8859 & 5771 & 5053 & 5968 & 4993 & 11 & Right Southern Elsont Ganabiat* & 1270 & 1067 & 1910 & 0 & 0 & 0 \\
\hline & & 3556 & 4462 & 3980 & 4737 & 5595 & 4681 & \multirow{3}{*}{12} & & 2921 & 3219 & 3006 & 2295 & 2443 & 2622 \\
\hline & & 1354 & 1624 & 1710 & 2562 & 3025 & 2531 & & Baheege & 1346 & 1122 & 1699 & 1515 & 1441 & 1711 \\
\hline & & 359 & 364 & 578 & 1404 & 1658 & 1387 & & & 775 & 604 & 1127 & 1093 & 774 & 1318 \\
\hline & & 5569 & 7237 & 6126 & 4969 & 27942 & 5411 & 12.1 & Abo Amera & 1312 & 1507 & 1695 & 811 & 673 & 1163 \\
\hline 3.1 & Left Elmaana & 3019 & 3771 & 3730 & 3639 & 19719 & 3962 & 12.2 & Elkhalifaa & 516 & 558 & 736 & 486 & 416 & 691 \\
\hline & & 693 & 767 & 1061 & 752 & 1941 & 924 & 13 & Bani Rezah & 1407 & 1389 & 1964 & 1048 & 690 & 1398 \\
\hline 3.1 .1 & Serage Banch & 562 & 496 & 982 & 1053 & 1255 & 1356 & 14 & Abnoub Branh & 525 & 445 & 937 & 1343 & 1224 & 1714 \\
\hline 3.1 .2 & Elfaiama Branch & 1435 & 1455 & 2314 & 1100 & 821 & 1829 & 15 & Elkadadeh Western Branch & 938 & 905 & 1423 & 837 & 594 & 1138 \\
\hline 3.2 & Amro Branch & 766 & 728 & 1284 & 1022 & 876 & 1472 & 16 & Bani Ibrahem Western Branch & 919 & 989 & 1430 & 720 & 468 & 1099 \\
\hline & & 1307 & 1377 & 1462 & 959 & 1067 & 1128 & 17 & Elsawalem Southern Branch & 677 & 666 & 1113 & 602 & 452 & 968 \\
\hline & Salebat Elmaasara & 2203 & 2161 & 2761 & 2243 & 2496 & 2638 & 18 & Elrawateb Branch & 375 & 344 & 641 & 358 & 260 & 583 \\
\hline 3.3 & Ganabia & 648 & 622 & 863 & 782 & 870 & 920 & 10 & Piobt Northern Flsont Ganobint & 4017 & 4426 & 4133 & 2761 & 2144 & 3134 \\
\hline & & 641 & 597 & 921 & 959 & 1067 & 1128 & 19 & Right Northern Elsont Ganabiat & 1596 & 1612 & 2032 & 1602 & 916 & 2004 \\
\hline 3.3 .1 & Elmanshia Branch & 393 & 347 & 687 & 492 & 307 & 757 & 19.1 & Elsihabia Branch & 1241 & 1441 & 1642 & 1066 & 946 & 1481 \\
\hline 3.31 & Elgamasea Branch & 670 & 637 & 1123 & 1106 & 1317 & 1424 & 19.2 & Asham Allah* & 287 & 273 & 481 & 0 & 0 & 0 \\
\hline 3.4 & Elqasr Branch & 364 & 346 & 610 & 701 & 780 & 903 & & & 508 & 536 & 501 & 368 & 331 & 385 \\
\hline 3.5 & Elquata Branch & 357 & 303 & 637 & 511 & 434 & 736 & 20 & Bani Mohamed & 3527 & 3563 & 3777 & 3204 & 2658 & 3559 \\
\hline 4 & Elghwaish Branch & 341 & 289 & 609 & 206 & 148 & 376 & & & 2273 & 2256 & 2650 & 2517 & 2254 & 2927 \\
\hline 5 & Elnabari Alwasta & 1287 & 1274 & 1736 & 2196 & 2911 & 2698 & 20.1 & Left Northern Elsont Ganabiat & 1258 & 1170 & 1806 & 876 & 749 & 1313 \\
\hline 6 & Boni Mor & 634 & 723 & 744 & 638 & 1018 & 671 & 20.2 & elmarawna & 469 & 453 & 711 & 347 & 246 & 549 \\
\hline 0 & Banı Mor & 543 & 505 & 779 & 843 & 1025 & 1006 & 20.3 & Sahel Elaqab & 572 & 569 & 841 & 521 & 309 & 789 \\
\hline 6.1 & Quernaw Branch & 537 & 417 & 834 & 390 & 292 & 649 & 20.4 & Abo Diab Branch & 610 & 619 & 983 & 491 & 364 & 804 \\
\hline 7 & Gazerat Bani Mor & 812 & 776 & 1133 & 851 & 708 & 1151 & 20.5 & Elmansora Western Branh & 300 & 275 & 513 & 369 & 282 & 559 \\
\hline 8 & Sahel Bani Mor & 1570 & 1504 & 2028 & 950 & 592 & 1356 & 20.6 & Sahel Bani Mohammed & 2602 & 2211 & 3426 & 2408 & 1962 & 2977 \\
\hline 8.1 & Elbaharwa & 341 & 289 & 609 & 175 & 82 & 327 & 21 & Shaqequel & 1222 & 1173 & 1628 & 807 & 609 & 1179 \\
\hline 0 & & 2834 & 3429 & 2785 & 3151 & 3519 & 2914 & 22 & Elmaabda Sothern Branch & 448 & 455 & 723 & 211 & 108 & 404 \\
\hline 9 & Elgharbia & 2233 & 2021 & 3053 & 4126 & 4875 & 4171 & 23 & Elmaabda Northern Branch & 1721 & 1841 & 2308 & 1444 & 509 & 1888 \\
\hline 9.1 & Hoshet Kom Aboshil & 433 & 367 & 773 & 196 & 135 & 327 & 24 & Elshikh Saed Branh & 1524 & 1472 & 2023 & 1991 & 1343 & 2351 \\
\hline 9.2 & Ali Bek & 670 & 637 & 1123 & 747 & 618 & 1173 & 24.1 & Abo Meshel & 289 & 245 & 515 & 313 & 214 & 502 \\
\hline 9.3 & Western Elnasara & 3080 & 3921 & 3764 & 1170 & 782 & 1727 & 24.2 & Sahel Elmaabda & 869 & 676 & 1351 & 931 & 618 & 1333 \\
\hline 9.4 & Eastern Elnasara & 2544 & 3300 & 3077 & 1472 & 1208 & 1968 & 25 & Emtedad Elsont & 1519 & 1652 & 1854 & 433 & 362 & 627 \\
\hline
\end{tabular}


Aswan University Journal of Environmental Studies (AUJES) 2 (3), pp. 190-201, (2021).

Online ISSN: 2735-4237, Print: ISSN 2735-4229. https://aujes.journals.ekb.eg/

Table 3: Meteorological data of El-Sont canal and its distributaries region, (Abu-Zeid, 2021)

\begin{tabular}{|c|c|c|c|c|c|c|c|}
\hline Month & $\begin{array}{c}\text { Max. } \\
\text { Temp. oC }\end{array}$ & $\begin{array}{c}\text { Min. } \\
\text { Temp. oC }\end{array}$ & $\begin{array}{c}\text { Avg. Temp. } \\
\text { oC }\end{array}$ & $\begin{array}{c}\text { \% RH } \\
\max \end{array}$ & $\begin{array}{c}\text { Pan Evaporation } \\
\mathrm{mm} / \text { day }\end{array}$ & $\begin{array}{c}\text { wind speed } \\
(\mathrm{km} / \mathrm{h})\end{array}$ & $\begin{array}{c}\text { No. of sunny } \\
\text { hours / day }\end{array}$ \\
\hline Jan & 18 & 6.50 & 22.40 & 60.30 & 2.80 & 16.00 & 8.90 \\
\hline Feb. & 22.5 & 11.20 & 26.10 & 52.60 & 3.20 & 17.30 & 9.70 \\
\hline Mar. & 25 & 14.20 & 30.50 & 42.90 & 4.40 & 19.80 & 9.90 \\
\hline Apr. & 29 & 17.10 & 35.10 & 36.50 & 6.40 & 21.30 & 10.30 \\
\hline May & 32 & 22.00 & 38.10 & 35.10 & 6.60 & 20.30 & 11.40 \\
\hline Jun. & 36 & 24.90 & 40.70 & 37.40 & 6.90 & 21.00 & 12.30 \\
\hline Jul. & 37 & 25.30 & 39.10 & 41.50 & 7.40 & 19.50 & 12.20 \\
\hline Aug. & 34.5 & 24.80 & 40.30 & 40.70 & 8.00 & 19.80 & 11.90 \\
\hline Sep. & 29 & 23.80 & 38.50 & 46.20 & 7.00 & 21.70 & 10.80 \\
\hline Oct. & 29 & 20.90 & 33.00 & 51.30 & 5.70 & 19.20 & 10.00 \\
\hline Nov. & 27 & 13.20 & 27.00 & 54.70 & 5.00 & 15.20 & 9.40 \\
\hline Dec. & 22.5 & 9.00 & 23.20 & 63.20 & 3.10 & 16.80 & 9.00 \\
\hline
\end{tabular}

Table 4: Evaporation losses for designed and existing water sections of El-Sont canal and its network

\begin{tabular}{|c|c|c|c|}
\hline \multirow{2}{*}{ No } & Canal Name & \multicolumn{2}{c|}{$\begin{array}{c}\text { Max. Evaporation } \\
\text { losses ( }\end{array}$} \\
\cline { 3 - 4 } & \multicolumn{2}{|c|}{ /day) } \\
\hline \multirow{2}{*}{$\mathbf{1}$} & El-Songned & Existing \\
\cline { 2 - 4 } & El-Sont (abnoub) & 3060 & 1021 \\
\hline $\mathbf{2}$ & Sothern Elnabary & 61 & 102 \\
\hline $\mathbf{3}$ & Elmaasara & 421 & 533 \\
\hline $\mathbf{3 . 1}$ & Left Elmaana Ganabia & 376 & 475 \\
\hline $\mathbf{3 . 1 . 1}$ & Serage Banch & 33 & 51 \\
\hline $\mathbf{3 . 1 . 2}$ & Elfaiama Branch & 77 & 90 \\
\hline $\mathbf{3 . 2}$ & Amro Branch & 43 & 65 \\
\hline $\mathbf{3 . 3}$ & Salebat Elmaasara Ganabia & 226 & 268 \\
\hline $\mathbf{3 . 3 . 1}$ & Elmanshia Branch & 23 & 27 \\
\hline $\mathbf{3 . 3 1}$ & Elgamasea Branch & 38 & 54 \\
\hline $\mathbf{3 . 4}$ & Elqasr Branch & 20 & 33 \\
\hline $\mathbf{3 . 5}$ & Elquata Branch & 22 & 30 \\
\hline $\mathbf{4}$ & Elghwaish Branch & 21 & 21 \\
\hline $\mathbf{5}$ & Elnabari Alwasta & 78 & 102 \\
\hline $\mathbf{6}$ & Bani Mor & 55 & 73 \\
\hline $\mathbf{6 . 1}$ & Quernaw Branch & 32 & 32 \\
\hline $\mathbf{7}$ & Gazerat Bani Mor & 46 & 51 \\
\hline $\mathbf{8}$ & Sahel Bani Mor & 93 & 78 \\
\hline $\mathbf{8 . 1}$ & Elbaharwa & 21 & 23 \\
\hline $\mathbf{9}$ & Elgharbia & 233 & 316 \\
\hline $\mathbf{9 . 1}$ & Hoshet Kom Aboshil & 26 & 17 \\
\hline $\mathbf{9 . 2}$ & Ali Bek & 38 & 52 \\
\hline $\mathbf{9 . 3}$ & Western Elnasara & 127 & 99 \\
\hline $\mathbf{9 . 4}$ & Eastern Elnasara & 104 & 93 \\
\hline $\mathbf{9 . 5}$ & Hablass & 116 & 132 \\
\hline
\end{tabular}

\begin{tabular}{|c|c|c|c|}
\hline \multirow[t]{2}{*}{ No } & \multirow[t]{2}{*}{ Canal Name } & \multicolumn{2}{|c|}{$\begin{array}{l}\text { Max. Evaporation } \\
\text { losses ( } \mathrm{m}^{3} / \text { day) }\end{array}$} \\
\hline & & Designed & Existing \\
\hline 9.6 & Hoshet Eltwabia & 65 & 106 \\
\hline 9.6 .1 & Eltwabia Southern Branch & 58 & 27 \\
\hline 10 & Kom Abo Shail Branch & 18 & 44 \\
\hline 11 & $\begin{array}{l}\text { Right Southern Elsont } \\
\text { Ganabiat* }\end{array}$ & 0 & 0 \\
\hline 12 & Baheege & 256 & 285 \\
\hline 12.1 & Abo Amera & 58 & 50 \\
\hline 12.2 & Elkhalifaa & 25 & 29 \\
\hline 13 & Bani Rezah & 70 & 90 \\
\hline 14 & Abnoub Branh & 32 & 70 \\
\hline 15 & Elkadadeh Western Branch & 49 & 66 \\
\hline 16 & Bani Ibrahem Western Branch & 47 & 57 \\
\hline 17 & Elsawalem Southern Branch & 37 & 45 \\
\hline 18 & Elrawateb Branch & 22 & 39 \\
\hline 19 & $\begin{array}{c}\text { Right Northern Elsont } \\
\text { Ganabiat }\end{array}$ & 263 & 236 \\
\hline 19.1 & Elsihabia Branch & 64 & 20 \\
\hline 19.2 & Asham Allah* & 0 & 0 \\
\hline 20 & Bani Mohamed & 305 & 331 \\
\hline 20.1 & $\begin{array}{c}\text { Left Northern Elsont } \\
\text { Ganabiat } \\
\end{array}$ & 65 & 64 \\
\hline 20.2 & Elmarawna & 25 & 25 \\
\hline 20.3 & Sahel Elaqab & 33 & 50 \\
\hline 20.4 & Abo Diab Branch & 33 & 37 \\
\hline 20.5 & Elmansora Western Branh & 17 & 25 \\
\hline 20.6 & Sahel Bani Mohammed & 150 & 157 \\
\hline 21 & Shaqequel & 60 & 58 \\
\hline 22 & Elmaabda Sothern Branch & 24 & 66 \\
\hline 23 & Elmaabda Northern Branch & 81 & 98 \\
\hline
\end{tabular}


Aswan University Journal of Environmental Studies (AUJES) 2 (3), pp. 190-201, (2021).

Online ISSN: 2735-4237, Print: ISSN 2735-4229. https://aujes.journals.ekb.eg/

\begin{tabular}{|c|c|c|c|c|c|c|c|}
\hline \multirow[t]{2}{*}{ No } & \multirow[t]{2}{*}{ Canal Name } & \multicolumn{2}{|c|}{$\begin{array}{c}\text { Max. Evaporation } \\
\text { losses (m }{ }^{3} / \text { day) }\end{array}$} & \multirow[t]{2}{*}{ No } & \multirow[t]{2}{*}{ Canal Name } & \multicolumn{2}{|c|}{$\begin{array}{l}\text { Max. Evaporation } \\
\text { losses ( } \mathrm{m}^{3} / \text { day) }\end{array}$} \\
\hline & & Designed & Existing & & & Designed & Existing \\
\hline 9.5 .1 & Elakrad Branch & 35 & 37 & 24 & Elshikh Saed Branh & 75 & 105 \\
\hline 9.5 .2 & Bani Zeid Branch & 41 & 69 & 24.1 & Abo Meshel & 18 & 25 \\
\hline 9.5.3 & Diab Branch & 16 & 12 & 24.2 & Sahel Elmaabda & 52 & 67 \\
\hline 9.5 .4 & Hablas Elgadida & 158 & 180 & 25 & Emtedad Elsont & 76 & 28 \\
\hline
\end{tabular}

*In the time of field trips, the canal was in off turn and not works.

\section{4- RESULTS AND DISCUSSIONS}

\section{1- Seepage losses}

Table 2 shows the values of the total seepage losses in the El-Sont canal and its network. From which, it is clear that:

- There are tangible differences in the values of seepage losses calculated by using the above most popular three equations. The Indian equation gives the biggest value, while Moritz's equation gives the lowest values, for El-Sont canal, and its network.

- There is a clear difference in the values of irrigation water losses through seepage that calculated from the existing real cross-sections on the ground and those calculated according to the dimensions obtained from the executive design sheets for those canals.

- The total values of seepage losses according to the dimensions obtained from the executive design sheets for El-Sont canal, and its off-taking canals, were about 213635, 261105, and $212144 \mathrm{~m}^{3} /$ day by using the Egyptian, Indian, and Moritz equations, respectively, with an average value of about $228962 \mathrm{~m}^{3} /$ day. While, using the dimensions of the existing on the ground sections, those values were about 210231, 298582, and $201515 \mathrm{~m}^{3} /$ day, with an average of about $236776 \mathrm{~m}^{3} /$ day. That is to say that, about $7814 \mathrm{~m}^{3} /$ day is lost only due to less care of maintaining the waterway dimensions on the ground exactly as it is in the executive design engineering sheets.

- The total amount of seepage losses from the designed sections of the El-Sont canal and its network, estimated by using the Egyptian, Indian, and Moritz's equations represents about $11.34 \%, 13.86 \%$, and $11.26 \%$, respectively, with an average of about $12.15 \%$ of the total discharge given at the head of the El-Sont canal.

- The total amount of seepage losses from the existing sections of the El-Sont canal and its network, estimated by using the Egyptian, Indian, and Moritz's equations represents about $11.16 \%, 15.85 \%$, and $10.70 \%$, with an average of about $12.66 \%$ from the total discharge that given at the head of the El-Sont canal, which is somewhat bigger than that percentage expected according to the dimensions taken from the official design sheets.

- The total lost water by seepage of the El-Sont canal and its network was estimated to be about 38.9 million cubic meters annually for the designed sections. While it was estimated to be about 39.54 million cubic meters for the dimensions of the existing sections. This difference is due to distortions and collapses in the water section, and internal side slopes of the canals. This significant amount of lost water will be saved when the rehabilitation national project is implemented and finished for the El-Sont canal and its network.

\section{2- Evaporation losses}

Table (4) shows the maximum calculated values of the total evaporation losses from the El-Sont canal and its network. From this table, it can be seen that: 
- The total values of evaporation losses from the designed sections of the El-Sont canal and its network are about $8681 \mathrm{~m}^{3} /$ day. While it was estimated for the existing sections with about $9480 \mathrm{~m}^{3} /$ day, with a difference reaches $800 \mathrm{~m}^{3} /$ day.

- The total amount of evaporation losses from the designed sections and the existing sections of the El-Sont canal and its network represent about $0.46 \%$, and $0.50 \%$ of the total discharge given at the head. This agrees with the study presented by Sahasrabudhe (2001) and Jadhav (2014).

- The values of evaporation losses for the existing sections are more than those calculated using the designed sections of the El-Sont canal network. This is due to the distortions of the water sections in some locations along the canal and its off-takings.

Based on the foregoing, the total average expected losses due to seepage and evaporation for the designed, and existing sections of the El-Sont canal and its network are estimated to be about $239,812 \mathrm{~m}^{3} /$ day, $248,628 \mathrm{~m}^{3} /$ day respectively, with an absolute average of about $244,220 \mathrm{~m}^{3} /$ day, at the season of maximum water needs. Most of these quantities of water will be saved by lining the canals with plain concrete, through the implementation of the national project of rehabilitation.

The amount of saved water in the case of lining with plain concrete was estimated using Davis Willson's equation (Leigh, 2014) to be about 36.31 million cubic meters annually, which is a significant value considering the water shortage faces Egypt at the present time.

The quantity of saved water, by the completion of the nowadays under implementation of the national rehabilitation project, can be sufficient to irrigate more than new 5000 acres using the modern irrigation systems, which equals about $15 \%$ of the total existing area irrigated now through EL-Sont canal and its off-takings.

\section{5- Conclusions}

The main conclusions of the current study can be summarized as follows:

(1) The study proved the high feasibility and the great economic, developmental, and environmental returns expected upon the completion of such a national project. In addition to the moral impact on the beneficiaries and farmers, which will raise the ceiling of their civilization and human aspirations.

(2) The estimated average seepage losses from the designed sections of the El-Sont canal and its network reaches about $228,962 \mathrm{~m}^{3} /$ day. This value represents $12.15 \%$ of the total discharge of the El-Sont canal, while it reaches about $248,628 \mathrm{~m}^{3} / \mathrm{day}$, which represents $12.66 \%$ if it is calculated for the existing on the ground cross-sections.

(3) The annual lost water by seepage only from the El-Sont canal and its network was estimated to be about 38.9, and 39.54 million cubic meters for the designed and existing sections, respectively.

(4) The total maximum evaporation losses from the existing and the designed sections of the El-Sont canal network were calculated to be about 9480 and $8681 \mathrm{~m}^{3} /$ day, respectively.

(5) The great quantities of saved or recovered water can be used for the reclamation and the irrigation of about 5000 new acres in the nearby area, representing about $15 \%$ of the existent_served area.

(6) The study, the field trips, the visits, and the personal direct communication with farmers and beneficiaries proved that the project had a great response to their hopes. Also, it 
improved their living standards, health, in addition to the environmental situation for all the Egyptian countryside.

\section{References}

Abu-zeid, T.S. (2021). Conveyance Losses Estimation for Open Channels in Middle Egypt, Case Study: Almanna Main Canal, and Its Branches., (JES) Journal of Engineering Sciences, 49(1), 64-84.

Ahuchaogu, I. E. (2015). Evaluation of Seepage Losses in an Earth Lined Canal: A Case Study of University of Uyo Farm. International Journal of Engineering Sciences and Management Research, 2(2), 2349-6193.

Akkuzu, E. (2012). Usefulness of Empirical Equations in Assessing Canal Losses through Seepage in Concrete-Lined Canal. Journal of Irrigation and Drainage Engineering, 138(5), 455-460.

Alam, M. M. (2004). Comparative Evaluation of Canal Seepage Investigation Techniques. Agricultural Water Management, 66(1), 65-76.

Allen, R. G., Pereira, L. S., Raes, D., \& Smith, M. (1998). Fao Irrigation and Drainage Paper No. 56. Rome,. Food and Agriculture Organization of the United Nations, 56 (97), E156.

Awan, A. A. (2017). Optimum Length of Lining to Reduce Losses in Watercourses by Using Advanced Non Linear Modelling. Doctoral Dissertation.

El Gamal, T. and. N. Zaki (2017). Egyptian Irrigation after the Aswan High Dam. Irrigated Agriculture in Egypt. (Pp. 47-79). Springer, Cham.

Farouk, M. (1979). Conveyance Losses in Canals. Conference on Water Resources Planning in Egypt, Cairo.

Jadhav, P. B. (2014). Conveyance Efficiency Improvement through Canal Lining and Yield Increment by Adopting Drip Irrigation in Command Area. International Journal of Innovative Research in Science, Engineering and Technology, 3 (4), 120-129.

Kacimov, A. R. (1992). Seepage Optimization for Trapezoidal Channel. Journal of Irrigation and Drainage Engineering, 118(4), 520-526.

Khan, A. K. (2015). Relationship between Seepage and Discharge for Kabul River in District Nowshera. Pakistan Journal of Agriculture, Agricultural Engineering and Veterinary Sciences, 31(2), 249-259.

Kraatz, D.B. (1977). Irrigation Canal Lining. Fao Land and Water Development Series No. 1, $18-50$.

Leigh, E. (2014). Evaluation of Methods for Predicting Seepage Loss Rates for the Hard Lined Irrigation Canals of the Lower Rio Grande Valley of Texas. Doctoral Dissertation.

Liu, S. W. (2016). Estimating The Evaporation from Irrigation Canals in Northwestern China Using the Double-Deck Surface Air Layer Model. Advances in Meteorology. Volume 2016 |Article ID 3670257 | https://doi.org/10.1155/2016/3670257

Martin, C. A. (2015). Uncertainty in Measuring Seepage from Earthen Irrigation Canals Using the Inflow-Outflow Method and in Evaluating the Effectiveness of Polyacrylamide Applications for Seepage Reduction. Doctoral Dissertation, Colorado State Universit.

Mowafy (2001). Seepage Losses in Ismailia Canal.. Sixth International Water Technology Conference, Iwtc, March, (Pp. 195-211). Alexandria, Egypt. 
MWRI (2020). Minsiatry of Water Resources and Irrigation,. Assiut Departement.

Robinson, A. R., and Rohwer, C. (1957). Measurement of Canal Seepage. Transactions of the American Society of Civil Engineers Paper No. 2865.

Sahasrabudhe, S. K. (2011). Irrigation Engineering and Hydraulic Structures. Publisher of Engineering \& Compute Books, 3rd Edition.

Shaikh, I. A. (2016). Estimating Earthen Tertiary Water Channel Seepage Losses as A Function of Soil Texture. Journal of Hydrologic Engineering, 21(2), 06015012.

Swamee, P. K. (2000). Design of Minimum Seepage Loss Canal Sections. Journal of Irrigation and Drainage Engineering, 126(1), 28-32.

Wang, W. X. (2019).Estimating Evaporation from Irrigation Canals in The Midstream Areas of the Heihe River Basin by A Double-Deck Surface Air Layer (Dsal) Model. Water, 11(9), 1788 .

Zhang, Q. C. (2017). Investigation of Irrigation Canal Seepage Losses through Use of Four Different Methods in Hetao Irrigation District, China. Journal of Hydrologic Engineering, 22(3), 05016035. 